RIMS-1734

Dual Consistent Systems of Linear Inequalities and Cardinality Constrained Polytopes

By

Satoru FUJISHIGE and Jens MASSBERG

December 2011

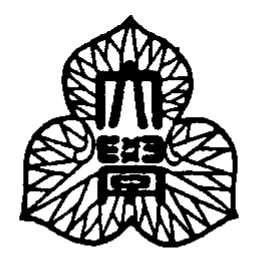

京都大学 数理解析研究所

RESEARCH INSTITUTE FOR MATHEMATICAL SCIENCES KYOTO UNIVERSITY, Kyoto, Japan 


\title{
Dual Consistent Systems of Linear Inequalities and Cardinality Constrained Polytopes
}

\author{
Satoru Fujishige* Jens Maßberg ${ }^{\dagger}$
}

December 6, 2011

\begin{abstract}
We introduce a concept of dual consistency of systems of linear inequalities with full generality. We show that a cardinality constrained polytope is represented by a certain system of linear inequalities if and only if the systems of linear inequalities associated with the cardinalities are dual consistent. Typical dual consistent systems of inequalities are those which describe polymatroids, generalized polymatroids, and dual greedy polyhedra with certain choice functions. We show that the systems of inequalities for cardinality-constrained ordinary bipartite matching polytopes are not dual consistent in general, and give additional inequalities to make them dual consistent. Moreover, we show that ordinary systems of inequalities for the cardinalityconstrained (poly)matroid intersection are not dual consistent, which disproves a conjecture of Maurras, Spiegelberg, and Stephan about a linear representation of the cardinality-constrained polymatroid intersection.
\end{abstract}

\section{Introduction}

Cardinality constrained polyhedra and their linear representations were first investigated by Maurras [7] and Camion and Maurras [1], and later rediscovered by Grötschel [5] for what is called a cardinality homogeneous set system (also see related recent work by

${ }^{*}$ Research Institute for Mathematical Sciences, Kyoto University, Kyoto 606-8502, Japan E-mail: fujishig@kurims.kyoto-u.ac.jp Research partly supported by a Grant-in-Aid from the Ministry of Education, Culture, Sports and Technology of Japan.

${ }^{\dagger}$ Research Institute for Discrete Mathematics, University of Bonn, Lennéstraße 2, 53111 Bonn, Germany E-mail: massberg@or.uni-bonn.de Research partly supported due to the "General Agreement for Cooperation between Hausdorff Center for Mathematics, University of Bonn and Research Institute for Mathematical Sciences, Kyoto University”. 
Kaibel and Stephan [6], Stephan [10], Maurras and Stephan [9], and Maurras, Spiegelberg, and Stephan [8]).

Given a finite nonempty set $S$, a combinatorial optimization problem $\Pi$ on $S$, and an increasing sequence $c=\left(c_{1}, \ldots, c_{m}\right)$ of nonnegative integers $c_{i}(i=1, \ldots, m)$, the cardinality constrained version $\Pi_{c}$ of $\Pi$ has the set of feasible solutions consisting of all feasible solutions of the original problem with the property that the cardinality (i.e. the number of elements) of every solution is equal to $c_{i}$ for some $i \in\{1, \ldots, m\}$. In $[7,1,5]$ they introduced forbidden cardinality inequalities of the form

$$
\begin{gathered}
\left(c_{p+1}-c_{p}\right) x(U)-\left(|U|-c_{p}\right) x(S) \leq c_{p}\left(c_{p+1}-|U|\right) \\
\text { for all } U \subseteq S \text { with } c_{p}<|U|<c_{p+1} \text { for some } p \in\{1, \ldots, m\},
\end{gathered}
$$

where $x(U)=\sum_{u \in U} x(u)$ for $U \subseteq S$, and showed that the inequalities hold for $\Pi_{c}$. Usually these inequalities are not facet-defining for the polyhedron associated with $\Pi_{c}$.

Recently Maurras and Stephan [9] derived strong valid inequalities that give a complete linear description for cardinality constrained matroids. This result has been generalized by Maurras, Spiegelberg, and Stephan $[8,11]$ to cardinality constrained polymatroids as follows. Given a polymatroid rank function $f: 2^{S} \rightarrow \mathbb{R}$ and an increasing sequence $\left(c_{1}, \ldots, c_{m}\right)$ of nonnegative integers $c_{i}(i=1, \ldots, m)$, they aim for the convex hull of all vectors $x$ of the polymatroid associated with $f$ of cardinality $c_{i}$ for some $i \in\{1, \ldots, m\}$, i.e. $x(S)=c_{i}$. The cardinality constrained polymatroid is shown to be determined by the following system of inequalities:

$$
\begin{aligned}
& x(U) \leq f(U) \quad(U \subseteq S), \\
& \left(c_{p+1}-c_{p}\right) x(U)-\left(f(U)-c_{p}\right) x(S) \leq c_{p}\left(c_{p+1}-f(U)\right) \\
& \quad\left(U \subseteq S \text { with } c_{p}<f(U)<c_{p+1} \text { for some } p \in\{1, \ldots, m-1\}\right), \\
& c_{1} \leq x(S) \leq c_{m}, \quad x \geq \mathbf{0} .
\end{aligned}
$$

In the present paper we introduce the concept of dual consistent systems of linear inequalities and formulate the cardinality constrained problem in a more general setting. In Section 2 we give a characterization of certain complete systems of linear inequalities expressing cardinality constrained polytopes with two cardinalities, where an essential role is played by the concept of dual consistency of systems of linear inequalities that we introduce in the present paper. Section 3 is concerned with multiple cardinality constraints. In Section 4 we show how the inequalities given in $[9,8,11]$ are derived from our result. We also show that the systems of inequalities for the cardinality-constrained ordinary bipartite matching polytopes and for the cardinality-constrained (poly)matroid intersection are not dual consistent in general. The latter implies that a conjecture of Maurras, Spiegelberg, and Stephan $[8,11]$ about a linear representation of the cardinality-constrained intersection of polymatroids does not hold in general. 


\section{Cardinality Constrained Polytopes}

In this section we consider the case where we have two cardinalities $c_{1}<c_{2}$ (i.e., $m=2$ ). The multiple cardinality case (i.e. $m>2$ ) will be discussed in Section 3.

\subsection{Dual Consistent Systems of Inequalities}

Let $S$ be a finite nonempty set and $\mathcal{Z}$ be a finite nonempty set of non-zero vectors in $\mathbb{R}^{S}$. Choose and fix a vector $z_{0} \in \mathcal{Z}$. Then, consider two functions $f_{i}: \mathcal{Z} \rightarrow \mathbb{R}(i=1,2)$ with $c_{1}:=f_{1}\left(z_{0}\right)<f_{2}\left(z_{0}\right)=: c_{2}$. Note that for the cardinality constrained polymatroid, $\mathcal{Z}$ is the set of characteristic vectors $\chi_{X}$ of all nonempty subsets $X$ of $S$ and $z_{0}$ is given by $\chi_{S}$, the all-one vector in $\mathbb{R}^{S}$. (For each $U \subseteq S$ the characteristic vector $\chi_{U} \in \mathbb{R}^{S}$ is defined by $\chi_{U}(u)=1$ for $u \in U$ and $\chi_{U}(u)=0$ for $u \in S \backslash U$.)

For each $i=1,2$ define the polyhedron

$$
P_{f_{i}}^{c_{i}}=\left\{x \in \mathbb{R}^{S} \mid \forall z \in \mathcal{Z}:\langle z, x\rangle \leq f_{i}(z),\left\langle z_{0}, x\right\rangle=c_{i}\right\},
$$

where $\langle\cdot, \cdot\rangle$ denotes the canonical inner product defined by $\langle z, x\rangle=\sum_{u \in S} z(u) x(u)$. We assume that the $P_{f_{i}}^{c_{i}}(i=1,2)$ are nonempty and bounded. $P_{f_{i}}^{c_{i}}$ can be regarded as a polyhedron restricted to vectors of cardinality $c_{i}$ where the cardinality of a vector $x$ is given by $\left\langle z_{0}, x\right\rangle$ (we may have $z_{0}=\chi_{S}$ (the all-one vector) in the ordinary case).

We are interested in obtaining a complete system of linear inequalities for the convex hull of $P_{f_{1}}^{c_{1}} \cup P_{f_{2}}^{c_{2}}$. To this end we introduce a concept of dual consistent systems of inequalities. We will show that if, and only if, the systems of linear inequalities appearing in (3) for $i=1,2$ are dual consistent, the convex hull is represented by the inequalities

$$
\begin{aligned}
& \left(c_{2}-c_{1}\right)\langle z, x\rangle-\left(f_{2}(z)-f_{1}(z)\right)\left\langle z_{0}, x\right\rangle \leq c_{2} f_{1}(z)-c_{1} f_{2}(z) \quad(z \in \mathcal{Z}), \\
& c_{1} \leq\left\langle z_{0}, x\right\rangle \leq c_{2}
\end{aligned}
$$

(see Theorem 1 to be shown below).

Remark 1: It should be noted that for each $i=1,2$, if we add the constraint $\left\langle z_{0}, x\right\rangle=c_{i}$ to (4), the system of inequalities (4) together with the added constraint is equivalent to

$$
\langle z, x\rangle \leq f_{i}(z) \quad(z \in \mathcal{Z}), \quad\left\langle z_{0}, x\right\rangle=c_{i} .
$$

This is exactly the system of inequalities defining $P_{f_{i}}^{c_{i}}$ in (3).

Now, for any $w \in \mathbb{R}^{S}$ and $i=1,2$ consider the following problem

$$
\begin{array}{lll}
\left(\mathbf{P}_{i}^{w}\right) & \text { Maximize } & \langle w, x\rangle \\
& \text { subject to } & x \in P_{f_{i}}^{c_{i}}
\end{array}
$$


Let $\hat{x}_{i}$ be an optimal solution of Problem $\left(\mathbf{P}_{i}^{w}\right)$ for $i=1,2$ and define

$$
\mathcal{Z}_{i}\left(\hat{x}_{i}\right)=\left\{z \in \mathcal{Z} \mid\left\langle z, \hat{x}_{i}\right\rangle=f_{i}(z)\right\} \quad(i=1,2),
$$

which represents the set of active (or tight) constraints of (6) at $\hat{x}_{i}$ for $i=1,2$. For each $i=1,2$ a set $\mathcal{B} \subseteq \mathcal{Z}$ is called a dual optimal base for Problem $\left(\mathbf{P}_{i}^{w}\right)$ if there exists an optimal solution $\hat{x}_{i}$ of Problem $\left(\mathbf{P}_{i}^{w}\right)$ such that

$$
\begin{aligned}
& \mathcal{B} \subseteq \mathcal{Z}_{i}\left(\hat{x}_{i}\right), \\
& \operatorname{rank} \mathcal{B}=|S|,
\end{aligned}
$$

where rank $\mathcal{B}$ is the rank of the matrix formed by the vectors in $\mathcal{B}$.

By definition $\hat{x}_{i}$ is an extreme point of $P_{f_{i}}^{c_{i}}$. It follows from (9) and (10) that $\hat{x}_{i}$ is a unique solution of the system of equations

$$
\langle z, x\rangle=f_{i}(z) \quad(z \in \mathcal{B}) .
$$

We assume that for every dual optimal base $\mathcal{B}$ appearing in the following arguments we have $z_{0} \in \mathcal{B}$. The systems of linear inequalities (6) for $i=1,2$ are called dual consistent if for every $w \in \mathbb{R}^{S}$ there exists a common dual optimal base $\mathcal{B}$ for $\left(\mathbf{P}_{1}^{w}\right)$ and $\left(\mathbf{P}_{2}^{w}\right)$. If there is no possibility of confusion, we also simply call the pair $\left(f_{1}, f_{2}\right)$ dual consistent in the sequel. Recall that the dual consistency depends on the choice of $c_{i}(i=1,2)$ and $z_{0}$ besides $f_{i}(i=1,2)$.

Examples: If $f_{1}$ and $f_{2}$ are submodular functions on $2^{S}$ with $f_{1}(S)=c_{1}<c_{2}=f_{2}(S)$ and $f_{1}(\emptyset)=f_{2}(\emptyset)=0$, the pair $\left(f_{1}, f_{2}\right)$ is dual consistent due to the greedy algorithm ([2] and also see, e.g., [4]). More generally, dual greedy polyhedra with a common choice function give us a dual consistent pair. This follows directly by their definitions (see [3]).

Remark 2: We have assumed that $P_{f_{i}}^{c_{i}}(i=1,2)$ are nonempty and bounded. We can extend the concept of dual consistency to systems of linear inequalities such that $P_{f_{i}}^{c_{i}}$ $(i=1,2)$ are pointed and have a common characteristic cone, by considering only weight vectors $w$ that give finite optimal values for Problem $\left(\mathbf{P}_{i}^{w}\right)$. (We may also call $\left(f_{1}, f_{2}\right)$ totally dual consistent with respect to $z_{0}$ if $\left(f_{1}, f_{2}\right)$ is dual consistent for $z_{0}$ and every choice of $c_{i}(i=1,2)$ such that $P_{f_{i}}^{c_{i}} \neq \emptyset(i=1,2)$. Polymatroids give typical examples of totally dual consistent systems with respect to the all-one vector as $z_{0}$.)

\subsection{The Convex-hull Polyhedron}

Define the polyhedron (polytope) $\hat{P}$ by

$$
\begin{aligned}
& \left(c_{2}-c_{1}\right)\langle z, x\rangle-\left(f_{2}(z)-f_{1}(z)\right)\left\langle z_{0}, x\right\rangle \leq c_{2} f_{1}(z)-c_{1} f_{2}(z) \quad(z \in \mathcal{Z}), \\
& c_{1} \leq\left\langle z_{0}, x\right\rangle \leq c_{2} .
\end{aligned}
$$


(Recall Remark 1 given in Section 2.1.)

Let $P_{f_{1}, f_{2}}^{c_{1}, c_{2}}$ denote the convex hull of $P_{f_{1}}^{c_{1}} \cup P_{f_{2}}^{c_{2}}$. We will show that $P_{f_{1}, f_{2}}^{c_{1}, c_{2}}=\hat{P}$ (defined by (12) and (13)) if and only if the pair $\left(f_{1}, f_{2}\right)$ is dual consistent. Before we show this, we analyze another (infinite) system of linear inequalities.

Denoting the optimal objective function value of (7) by $\zeta_{i}^{w}$, we introduce the condition that every $x \in \hat{P}$ satisfies

$$
\left(c_{2}-c_{1}\right)\langle w, x\rangle-\left(\zeta_{2}^{w}-\zeta_{1}^{w}\right)\left\langle z_{0}, x\right\rangle \leq c_{2} \zeta_{1}^{w}-c_{1} \zeta_{2}^{w} \quad\left(\forall w \in \mathbb{R}^{S}\right) .
$$

Lemma 1: If for all $w \in \mathbb{R}^{S}$ and all $x \in \hat{P}$ inequality (14) holds, then the pair $\left(f_{1}, f_{2}\right)$ is dual consistent.

(Proof) If we consider $\zeta_{i}^{w}$ for each $i=1,2$ as a function in $w \in \mathbb{R}^{S}$, it is what is called the support function of polytope $P_{f_{i}}^{c_{i}}$. Hence, the inequalities of (14) together with $c_{1} \leq$ $\left\langle z_{0}, x\right\rangle \leq c_{2}$ determine the convex hull $P_{f_{1}, f_{2}}^{c_{1}, c_{2}}$ of $P_{f_{1}}^{c_{1}} \cup P_{f_{2}}^{c_{2}}$. (Note that inequalities of (14) are exactly those which support both $P_{f_{i}}^{c_{i}}(i=1,2)$.) It follows from the assumption that $\hat{P} \subseteq P_{f_{1}, f_{2}}^{c_{1}, c_{2}}$. We can easily see that the converse inclusion relation also holds true, due to Remark 1. Consequently,

$$
\hat{P}=P_{f_{1}, f_{2}}^{c_{1}, c_{2}} .
$$

Since it suffices to consider an arbitrary generic $w \in \mathbb{R}^{S}$ to show the dual consistency, it follows from (15) that for a generic $w \in \mathbb{R}^{S}$ the unique optimal solutions $\hat{x}_{i}$ for problem $\mathbf{P}_{i}^{w}(i=1,2)$ are adjacent vertices of $\hat{P}\left(=P_{f_{1}, f_{2}}^{c_{1}, c_{2}}\right)$, which implies that there exists a dual optimal base for problem $\mathbf{P}_{i}^{w}(i=1,2)$ for all $w$.

Remark 3: Here we do not need that $f_{1}$ and $f_{2}$ have a common domain $\mathcal{Z}$. For different domains of $f_{1}$ and $f_{2}$ the above proof is valid to show that constraint (14) implies the dual consistency of $\left(f_{1}, f_{2}\right)$.

Remark 4: As noted in the above proof, the inequalities of (14) together with inequalities $c_{1} \leq\left\langle z_{0}, x\right\rangle \leq c_{2}$ determine the convex hull $P_{f_{1}, f_{2}}^{c_{1}, c_{2}}$ of $P_{f_{1}}^{c_{1}} \cup P_{f_{2}}^{c_{2}}$. Since $P_{f_{1}, f_{2}}^{c_{1}, c_{2}}$ is a polytope, we need only a finite number of inequalities from (14) besides (13) to obtain a representation of $P_{f_{1}, f_{2}}^{c_{1}, c_{2}}$ by linear inequalities, but the number of required inequalities could be much larger than $|\mathcal{Z}|$. Note that every inequality of (14) gives a hyperplane that supports both $P_{f_{1}}^{c_{1}}$ and $P_{f_{2}}^{c_{2}}$, and vice versa.

Next we show the following lemma.

Lemma 2: For any dual consistent pair $\left(f_{1}, f_{2}\right)$ with $f_{1}\left(z_{0}\right)=c_{1}<c_{2}=f_{2}\left(z_{0}\right)$ the convex hull $P_{f_{1}, f_{2}}^{c_{1}, c_{2}}$ of $P_{f_{1}}^{c_{1}} \cup P_{f_{2}}^{c_{2}}$ is expressed by (12) and (13).

(Proof) Recall that $\hat{P}$ is the polytope defined by (12) and (13) and that $P_{f_{1}, f_{2}}^{c_{1}, c_{2}} \subseteq \hat{P}$. Suppose that $P_{f_{1}, f_{2}}^{c_{1}, c_{2}} \neq \hat{P}$. Then there exists an edge $L$ of $\hat{P}$ connecting a vertex $x_{1}$ and 
a vertex $x_{2}$ of $\hat{P}$ such that one of the two is a vertex of $P_{f_{1}}^{c_{1}}$ or of $P_{f_{2}}^{c_{2}}$ and that the other belongs to $\hat{P} \backslash P_{f_{1}, f_{2}}^{c_{1}, c_{2}}$. We assume without loss of generality that $x_{1}$ is a vertex of $P_{f_{1}}^{c_{1}}$ and $x_{2} \in \hat{P} \backslash P_{f_{1}, f_{2}}^{c_{1}, c_{2}}$.

Let $\langle w, x\rangle=b$ be a supporting hyperplane of $\hat{P}$ that defines the edge $L$. Then $x_{1}$ is the unique optimal solution of Problem $\left(\mathbf{P}_{1}^{w}\right)$. Let $y_{2}$ be an optimal solution of Problem $\left(\mathbf{P}_{2}^{w}\right)$. We can assume that $w$ is (generically) chosen so that $y_{2}$ is a unique optimal solution as well. Because of the dual consistency there exists a base $\mathcal{B}$ such that $\mathcal{B}$ is a dual optimal base for both problems $\left(\mathbf{P}_{i}^{w}\right)(i=1,2)$. It follows from Remark 1 that $x_{1}$ and $y_{2}$ lie on the line $L^{\prime}$ determined by the system of equations given by (12) for all $z \in \mathcal{B} \backslash\left\{z_{0}\right\}$, each of (12) for such $z$ holding with equality. It follows that $x_{2}$ must coincide with $y_{2}$, which contradicts the assumption on $x_{2}$.

Lemma 3: If $P_{f_{1}, f_{2}}^{c_{1}, c_{2}}=\hat{P}$, then inequalities (14) hold for all $x \in \hat{P}$.

(Proof) Since $P_{f_{1}, f_{2}}^{c_{1}, c_{2}}$ is determined by (14) and inequalities $c_{1} \leq\left\langle z_{0}, x\right\rangle \leq c_{2}$, the present theorem follows.

Now it follows from Lemmas 1, 2, and 3 that

Theorem 1: The following three statements are equivalent:

(a) We have $P_{f_{1}, f_{2}}^{c_{1}, c_{2}}=\hat{P}$.

(b) Inequalities (14) hold for all $x \in \hat{P}$.

(c) The pair $\left(f_{1}, f_{2}\right)$ is dual consistent.

Remark 5: When the domains of $f_{1}$ and $f_{2}$ are different and given by $\mathcal{Z}_{1}$ and $\mathcal{Z}_{2}$, we can always obtain a common domain $\mathcal{Z}_{1} \cup \mathcal{Z}_{2}$ by adding redundant constraints.

Remark 6: For any two polytopes $P_{1}$ and $P_{2}$ lying on two distinct parallel hyperplanes, let $z_{0}$ be a common normal vector of the hyperplanes, and let $\mathcal{Z}$ be a finite set of normal vectors of hyperplanes (linear inequalities) that define the convex hull $P$ of $P_{1} \cup P_{2}$, connecting the two polytopes. Then we get two functions $f_{i}: \mathcal{V} \rightarrow \mathbb{R}(i=1,2)$ such that $P_{i}=P_{f_{i}}^{c_{i}}$ with $f_{i}\left(z_{0}\right)=c_{i}(i=1,2)$ and the pair $\left(f_{1}, f_{2}\right)$ is dual consistent.

This means that the systems of inequalities for any such two polytopes can be made dual consistent by adding some redundant inequalities. (Also see Remark 4.) 


\section{Multiple Cardinality Constrained Polytopes}

In Section 2 we have considered cardinality constrained polytopes with only two cardinalities $c_{1}$ and $c_{2}$. In the multiple cardinality case where $m>2$ there are a finite sequence of cardinalities $\left(c_{1}, \ldots, c_{m}\right)$ with $c_{1}<c_{2}<\cdots<c_{m}$ and functions $f_{1}, \ldots, f_{m}: \mathcal{Z} \rightarrow \mathbb{R}$ with $f_{i}\left(z_{0}\right)=c_{i}(i=1, \ldots, m)$, where $S, \mathcal{Z}$, and $z_{0}$ are the same as those in Section 2. We assume that each pair of $f_{i}$ and $f_{i+1}$ is dual consistent for $i=1, \ldots, m-1$. It should be noted that the relation of dual consistency on such pairs is not an equivalence relation, and it is not transitive, in particular.

Again we consider nonempty polytopes $P_{f_{i}}^{c_{i}}(i=1, \ldots, m)$ defined as in (3) and aim for a linear inequality representation of the convex hull $P_{f_{1}, \ldots, f_{m}}^{c_{1}, \ldots, c_{m}}$ of $P_{f_{1}}^{c_{1}} \cup P_{f_{2}}^{c_{2}} \cup \cdots \cup P_{f_{m}}^{c_{m}}$.

In the most general case it will be hard to derive inequalities for the convex hull if the inequalities $\langle z, x\rangle \leq f_{i}(z)$ of (3) $(1 \leq i \leq m$ and $z \in \mathcal{Z})$ are not valid for all points $x \in P_{f_{1}, \ldots, f_{m}}^{c_{1}, \ldots, c_{m}}$ with $\left\langle z_{0}, x\right\rangle=c_{i}$ for every $i=1, \ldots, m$. Hence we assume

$$
P_{f_{1}, \ldots, f_{m}}^{c_{1}, \ldots, c_{m}} \cap\left\{x \in \mathbb{R}^{S} \mid\left\langle z_{0}, x\right\rangle=c_{i}\right\}=P_{f_{i}}^{c_{i}} \quad(i=1, \ldots, m) .
$$

We also assume

(T) each inequality in (3) $(i=1, \ldots, m)$ defines a face (or supports the polytope with equality).

Here (T) is the tightness condition for each $f_{i}$ and $c_{i}$. It should be noted that the tightness condition (T) is not required when $m=2$.

Remark 7: Let $P_{*} \subset \mathbb{R}^{S}$ be a polyhedron, $z_{0} \in \mathbb{R}^{S} \backslash\{\mathbf{0}\}, c_{1}<\cdots<c_{m}$ a sequence of cardinalities, and $P_{*}^{c_{i}}=P_{*} \cap\left\{x \in \mathbb{R}^{S} \mid\left\langle z_{0}, x\right\rangle=c_{i}\right\}$ (nonempty and bounded). Then there is a finite set $\mathcal{Z} \subset \mathbb{R}^{S} \backslash\{\mathbf{0}\}$ and functions $f_{i}: \mathcal{Z} \rightarrow \mathbb{R}(1 \leq i \leq m)$ such that $P_{*}^{c_{i}}=P_{f_{i}}^{c_{i}}$ for all $i=1, \ldots, m$. Due to the convexity of the polyhedron $P_{*}$ equations (16) hold true.

Under assumption (16) we immediately get

$$
P_{f_{1}, \ldots, f_{m}}^{c_{1}, \ldots, c_{m}}=\bigcup_{1 \leq i \leq m-1} \operatorname{Conv}\left(P_{f_{i}}^{c_{i}} \cup P_{f_{i+1}}^{c_{i+1}}\right)
$$

where $\operatorname{Conv}(\cdot)$ is the convex hull operator in $\mathbb{R}^{S}$.

We can easily generalize Theorem 1 to the multiple cardinality case as follows. Define a polyhedron (polytope) $\hat{P}_{f_{1}, \ldots, f_{m}}^{c_{1}, \ldots, c_{m}}$ by

$$
\begin{aligned}
& \left(c_{i+1}-c_{i}\right)\langle z, x\rangle-\left(f_{i+1}(z)-f_{i}(z)\right)\left\langle z_{0}, x\right\rangle \leq c_{i+1} f_{i}(z)-c_{i} f_{i+1}(z) \\
& (z \in \mathcal{Z}, i=1, \ldots, m-1) \text {, } \\
& c_{1} \leq\left\langle z_{0}, x\right\rangle \leq c_{m}
\end{aligned}
$$


For each $i=1, \ldots, m-1$ and $z \in \mathcal{Z} \backslash\left\{z_{0}\right\}$ denote the inequality in (18) by $H_{i}^{z}$. We see from the tightness condition (T) and Theorem 1 that inequality $H_{i}^{z}$ supports the following three polytopes:

$$
P_{f_{i}}^{c_{i}}, \quad P_{f_{i+1}}^{c_{i+1}}, \quad \operatorname{Conv}\left(P_{f_{i}}^{c_{i}} \cup P_{f_{i+1}}^{c_{i+1}}\right) .
$$

It follows from assumption (16) and the convexity of $P_{f_{1}, \ldots, f_{m}}^{c_{1}, \ldots, c_{m}}$ that inequality $H_{i}^{z}$ is also valid for other polytopes $P_{f_{j}}^{c_{j}}(j \in\{1, \ldots, m\} \backslash\{i, i+1\})$. It should be noted that (convex) polytopes $\operatorname{Conv}\left(P_{f_{i}}^{c_{i}} \cup P_{f_{i+1}}^{c_{i+1}}\right)(i=1, \ldots, m-1)$ and $P_{f_{1}, \ldots, f_{m}}^{c_{1}, \ldots, c_{m}}$ have the same dimension.

Because of this argument and Theorem 1 we then get

Theorem 2: Under assumption (16) and the tightness condition (T) the following statements are equivalent:

(i) We have $P_{f_{1}, \ldots, f_{m}}^{c_{1}, \ldots, c_{m}}=\hat{P}_{f_{1}, \ldots, f_{m}}^{c_{1}, \ldots, c_{m}}$. That is, the system of inequalities in (18) and (19) represents the cardinality constrained polytope $P_{f_{1}, \ldots, f_{m}}^{c_{1}, \ldots, c_{m}}$.

(ii) Functions $f_{i}$ and $f_{i+1}$ are dual consistent for all $i=1, \ldots, m-1$.

\section{Examples and Counterexamples}

\subsection{Polymatroids}

For each $U \subseteq S$ we identify $U$ with the characteristic vector $\chi_{U} \in \mathbb{R}^{S}$.

We now show how the forbidden cardinality inequalities of [9] and [11] can be derived from (12). To this end let $f: 2^{S} \rightarrow \mathbb{R}_{\geq 0}$ be a polymatroid rank function and let $\mathcal{Z}=$ $2^{S} \backslash\{\emptyset\}$ and $z_{0}=S$. Also let $0 \leq c_{1}<\cdots<c_{m} \leq f(S)$. Now define functions $f_{i}: \mathcal{Z} \cup\{\emptyset\} \rightarrow \mathbb{R}(i=1, \ldots, m)$ by $f_{i}(U)=\min \left\{c_{i}, f(U)\right\}$ for $U \in \mathcal{Z} \cup\{\emptyset\}$. Consider polytopes $P_{f_{i}}^{c_{i}}$ defined by (3) for all $i=1, \ldots, m$.

Note that for each $i=1, \ldots, m \quad f_{i}$ is the rank function of the truncation, by $c_{i}$, of the underlying polymatroid with rank function $f$. Due to the submodularity of $f_{i}$ $(i=1, \ldots, m)$, the functions $f_{i}$ and $f_{i+1}$ are dual consistent for all $i=1, \ldots, m-1$. Moreover, the tightness condition (T) holds for all $f_{i}$ and $c_{i}$ and (16) also holds.

Hence by Theorem 2 the system of inequalities in (18) and (19) defines the convex hull of $P_{f_{1}}^{c_{1}} \cup \cdots \cup P_{f_{m}}^{c_{m}}$. Note that Remark 7 applies to the current polymatroid case.

Inequalities (18) can be written as

$$
\begin{aligned}
\left(c_{i+1}-c_{i}\right) x(U)-\left(f_{i+1}(U)-f_{i}(U)\right) x(S) & \leq c_{i+1} f_{i}(U)-c_{i} f_{i+1}(U) \\
& (U \subseteq S, i=1, \ldots, m-1) .
\end{aligned}
$$

For any $i \in\{1, \ldots, m-1\}$ consider any subset $U \subseteq S$ such that $c_{i} \leq f(U) \leq c_{i+1}$. Then by definition of $f_{i}$ and $f_{i+1}$ we get $f_{i}(U)=c_{i}$ and $f_{i+1}(U)=f(U)$. Hence inequality 
(20) reduces to

$$
\left(c_{i+1}-c_{i}\right) x(U)-\left(f(U)-c_{i}\right) x(S) \leq c_{i}\left(c_{i+1}-f(U)\right)
$$

for such $U$. These are exactly the $f$-induced forbidden cardinality inequalities shown in $[8,9,11]$ (see (2)). It should be noted that if $c_{i+1}<f(U)$, (20) becomes $x(U) \leq f(U)$ and if $f(U)<c_{i}$, then $0 \leq x(S \backslash U)$, both being valid inequalities for the original polymatroid polytope. More precisely, (20) together with $c_{1} \leq x(S) \leq c_{m}$ implies (2).

\subsection{Bipartite Matchings}

Let $G=\left(V^{+}, V^{-} ; E\right)$ be a bipartite graph with a vertex bipartition $\left(V^{+}, V^{-}\right)$and a set $E$ of edges between $V^{+}$and $V^{-}$. For any vertex $v \in V^{+} \cup V^{-}$denote by $\delta v$ the set of edges incident to $v$.

Let $w$ be a weight vector generically chosen from $\mathbb{R}^{E}$ and $c_{i}(i=1,2)$ be positive integers with $c_{1}<c_{2}$ such that there exists at least one matching $M$ in $G$ of size $|M|=c_{2}$. Then for each $i=1,2$ consider a maximum-weight matching problem with a cardinality constraint, relaxed in $\mathbb{R}^{E}$ as follows.

$$
\begin{array}{lll}
\left(\mathbf{P}_{i}^{w}\right) \quad \text { Maximize } & \sum_{e \in E} w(e) x(e) \\
\text { subject to } & \sum_{e \in \delta v^{+}} x(e) \leq 1 \quad\left(v^{+} \in V^{+}\right), \\
& \sum_{e \in \delta v^{-}} x(e) \leq 1 \quad\left(v^{-} \in V^{-}\right), \\
& 0 \leq x(e) \leq 1 \quad(e \in E), \\
& \sum_{e \in E} x(e)=c_{i} .
\end{array}
$$

Here, we have $z_{0}=\chi_{E} \in \mathbb{R}^{E}$, and $\mathcal{Z}$ is the set of the coefficient vectors of the inequalities and the equation appearing in (22), where $0 \leq x(e)$ should be regarded as an inequality $-x(e) \leq 0$ for all $e \in E$. Also, for each $i=1,2$ function $f_{i}: \mathcal{Z} \rightarrow \mathbb{R}$ is defined so as to take the values specified by the right-hand sides of (22).

For each $i=1,2$ let $\hat{x}_{i}$ be the unique optimal solution of Problem $\left(\mathbf{P}_{i}^{w}\right)$, where the uniqueness is due to the choice of generic $w$. Then, due to the integrality of (22), for each $i=1,2$ there is a matching $M_{i} \subseteq E$ in $G$ such that $\hat{x}_{i}=\chi_{M_{i}}$.

Consider the symmetric difference $M_{1} \Delta M_{2} \equiv\left(M_{1} \backslash M_{2}\right) \cup\left(M_{2} \backslash M_{1}\right)$. Then $M_{1} \Delta M_{2}$ can be decomposed into vertex-disjoint paths and possible cycles. Note that such paths and cycles are formed by alternating edges of $M_{1}$ and $M_{2}$. Because of the uniqueness of the optimal solutions there does not exist any such alternating cycle or path of even length (even number of edges). Suppose that the vertex-disjoint paths are then given by $Q^{(k)}$ 
$(k=1, \ldots, \ell)$, each of which satisfies one of the following two. We denote by $E\left(Q^{(k)}\right)$ the edge set of $Q^{(k)}$.

$$
\begin{aligned}
& \left|M_{2} \cap E\left(Q^{(k)}\right)\right|=\left|M_{1} \cap E\left(Q^{(k)}\right)\right|+1, \\
& \left|M_{2} \cap E\left(Q^{(k)}\right)\right|=\left|M_{1} \cap E\left(Q^{(k)}\right)\right|-1 .
\end{aligned}
$$

Let $n_{+}$and $n_{-}$, respectively, be the number of paths $Q^{(k)}$ of type (23) and that of type (24). Then we see that $\ell=n_{+}+n_{-}$and $n_{+}-n_{-}=c_{2}-c_{1} \geq 1$. Suppose that $n_{-} \geq 1$, and then consider a pair of a path of type (23) and a path of type (24). The pair contains the same number of arcs from $M_{1}$ and from $M_{2}$ in total, which contradicts the uniqueness of the optimal solutions. It follows that we have $n_{-}=0$, i.e., $n_{+}=c_{2}-c_{1}=\ell$.

For each path $Q^{(k)}$ denote by $\tilde{V}\left(Q^{(k)}\right)$ the set of intermediate (inner) vertices of $Q^{(k)}$, its initial and terminal vertices being discarded.

The tight inequalities (equations) in (22) common for $i=1,2$ are given as follows.

(i) For all $e \in M_{1} \cap M_{2}$ we have $x(e)=1$.

(ii) For all $e \in E \backslash\left(M_{1} \cup M_{2}\right)$ we have $x(e)=0$.

(iii) For each $k=1, \ldots, c_{2}-c_{1}$, associated with (23), we have

$$
\begin{array}{ll}
\sum_{e \in \delta v^{+}} x(e)=1 & \left(v^{+} \in \tilde{V}\left(Q^{(k)}\right) \cap V^{+}\right), \\
\sum_{e \in \delta v^{-}} x(e)=1 & \left(v^{-} \in \tilde{V}\left(Q^{(k)}\right) \cap V^{-}\right) .
\end{array}
$$

For each $k=1, \ldots, c_{2}-c_{1}$ the total number of equations appearing in (25) and (26) is equal to $\left|\tilde{V}\left(Q^{(k)}\right)\right|=\left|E\left(Q^{(k)}\right)\right|-1$.

Since equations of type (i) $x(e)=0$ and type (ii) $x(e)=1$ can always be taken into a dual base, we delete the arcs of $\left(M_{1} \cap M_{2}\right) \cup\left(E \backslash\left(M_{1} \cup M_{2}\right)\right)$ from $G$, and assume that $M_{1} \cap M_{2}=\emptyset$ and $E=M_{1} \cup M_{2}$ in the sequel.

If $c_{2}-c_{1}=1$, then the symmetric difference $M_{1} \Delta M_{2}$ must form a single path. We can see that the system of exactly $|E|$ equations of (i), (ii), and (iii) (with $c_{2}-c_{1}=1$ ) together with the cardinality constraint uniquely determines the optimal solution $\hat{x}_{i}$ for each $i=1,2$. Hence $\left(\mathbf{P}_{i}^{w}\right)(i=1,2)$ have a common optimal dual base. It follows that the systems of inequalities for $\left(\mathbf{P}_{i}^{w}\right)(i=1,2)$ is dual consistent. In the present case the cardinality constrained polytope is represented by (22) with the last equation being replaced by

$$
c_{1} \leq \sum_{e \in E} x(e) \leq c_{2}\left(=c_{1}+1\right)
$$

The present fact is closely related to the primal-dual, augmenting path algorithm for the maximum-weight matching problem, and is well known. 
On the other hand, if $c_{2}-c_{1} \geq 2$, there are $c_{2}-c_{1}$ (at least two) paths of (23), so that the number of tight equations common for $i=1,2$ is at most $|E|-2$. Hence $\left(\mathbf{P}_{i}^{w}\right)(i=1,2)$ cannot have any common dual optimal base even if we take the cardinality constraint into account. That is, the systems of inequalities for $\left(\mathbf{P}_{i}^{w}\right)(i=1,2)$ are not dual consistent. This implies that we need some additional redundant inequalities for $\left(\mathbf{P}_{i}^{w}\right)(i=1,2)$ to express the cardinality constrained polytope $P_{f_{1}, f_{2}}^{c_{1}, c_{2}}=\operatorname{Conv}\left(P_{f_{1}}^{c_{1}} \cup P_{f_{2}}^{c_{2}}\right)$. Such additional inequalities can be given in a form of (14). A set of additional inequalities is, for example, given as follows.

For each $k=1, \ldots, c_{2}-c_{1}$ let $e^{(k)}$ be an edge of $M_{2}$ in path $Q^{(k)}$. For any $F \subseteq E$ let $\sigma(F)$ be the maximum size of a matching in $G$ contained in $F$. Put $M:=M_{1} \cup M_{2}$. By construction we have $\sigma(M)=\left|M_{2}\right|=c_{2}$. For each $k=1, \ldots, c_{2}-c_{1}$ consider set $M \backslash\left\{e^{(k)}\right\}$. We see that $M_{2} \backslash\left\{e^{(k)}\right\}$ is a matching in $M \backslash\left\{e^{(k)}\right\}$ and there cannot be a larger one within $M \backslash\left\{e^{(k)}\right\}$. Hence we have $\sigma\left(M \backslash\left\{e^{(k)}\right\}\right)=c_{2}-1$.

It follows that each inequality

$$
\sum_{e \in M \backslash\left\{e^{(k)}\right\}} x(e) \leq c_{1}
$$

is valid for $\left(\mathbf{P}_{1}^{w}\right)$ and is tight for $x=\chi_{M_{1}}$, while each inequality

$$
\sum_{e \in M \backslash\left\{e^{(k)}\right\}} x(e) \leq c_{2}-1
$$

is valid for $\left(\mathbf{P}_{2}^{w}\right)$ and is tight for $x=\chi_{M_{2}}$. Note that inequalities (28) (or (29)) together with the other tight inequalities (25) and (26) are linearly independent since $c_{2}-c_{1} \geq 2$. (One of these inequalities can be deleted, if we take into account the cardinality constraint $x(E)=c_{i}$ for $i=1$ or 2.) Adding inequalities (28) to $\left(\mathbf{P}_{1}^{w}\right)$ and (29) to $\left(\mathbf{P}_{2}^{w}\right)$, we have a common dual optimal base formed by these inequalities.

Any generic weight $w$ determines a pair of optimal matchings $M_{1}$ for $\left(\mathbf{P}_{1}^{w}\right)$ and $M_{2}$ for $\left(\mathbf{P}_{2}^{w}\right)$. Let us call such a pair $\left(M_{1}, M_{2}\right)$ an admissible pair. Then, adding inequalities (28) to $\left(\mathbf{P}_{1}^{w}\right)$ and (29) to $\left(\mathbf{P}_{2}^{w}\right)$ for all admissible pairs $\left(M_{1}, M_{2}\right)$ makes the systems of inequalities for $\left(\mathbf{P}_{i}^{w}\right)(i=1,2)$ dual consistent, i.e., it makes them have a common dual base for any $w$. It should be noted that for a non-generic $w$, even if optimal matchings $M_{1}$ and $M_{2}$ are not unique, we can always find optimal matchings $M_{1}^{\prime}$ and $M_{2}^{\prime}$ with $\left|M_{1}^{\prime}\right|=c_{1}$ and $\left|M_{2}^{\prime}\right|=c_{2}$ such that $\left(M_{1}^{\prime}, M_{2}^{\prime}\right)$ is admissible.

\subsection{Matroid intersection}

Suppose we are given two matroids $\mathbf{M}^{(1)}$ and $\mathbf{M}^{(2)}$ on a ground set $S$ with rank functions $r_{1}$ and $r_{2}$, respectively. Define the function $f: 2^{S} \rightarrow \mathbb{R}$ by

$$
f(U)=\min \left\{r_{1}(T)+r_{2}(U \backslash T) \mid T \subseteq U\right\} \quad(\forall U \subseteq S) .
$$


Note that $f(U)$ is equal to the maximum size of a common independent set of $\mathbf{M}^{(1)}$ and $\mathbf{M}^{(2)}$ restricted on $U \subseteq S$. Consider the matroid intersection polytope represented by

$$
\begin{aligned}
& x(U) \leq f(U) \quad(U \subseteq S), \\
& x \geq \mathbf{0} .
\end{aligned}
$$

Taking into account the nonnegativity constraint, define

$$
\begin{aligned}
& \mathcal{Z}=\mathcal{Z}_{a} \cup \mathcal{Z}_{b} \\
& \quad \mathcal{Z}_{a}=\left\{\chi_{U} \mid U \subseteq S, U \neq \emptyset\right\}, \quad \mathcal{Z}_{b}=\left\{-\chi_{e} \mid e \in S\right\} .
\end{aligned}
$$

Let $c_{1}$ and $c_{2}$ with $c_{1}<c_{2} \leq f(S)$ be two given positive integers (the cardinalities) and define $f_{i}: \mathcal{Z} \rightarrow \mathbb{R}$ for each $i=1,2$ by

$$
f_{i}(z)=\left\{\begin{array}{ll}
\min \left\{f(U), c_{i}\right\} & \left(z=\chi_{U}, \emptyset \neq U \subseteq S\right) \\
0 & \left(z=-\chi_{e}, e \in S\right)
\end{array} \quad(\forall z \in \mathcal{Z}) .\right.
$$

The cardinality-constrained polytopes $P_{f_{1}}^{c_{1}}$ and $P_{f_{2}}^{c_{2}}$ are given by (3).

Let us examine whether the pair $\left(f_{1}, f_{2}\right)$ is dual consistent in general, i.e. whether the convex hull of $P_{f_{1}}^{c_{1}} \cup P_{f_{2}}^{c_{2}}$ is described by (4) and (5):

$$
\begin{aligned}
& \left(c_{2}-c_{1}\right)\langle z, x\rangle-\left(f_{2}(z)-f_{1}(z)\right)\left\langle z_{0}, x\right\rangle \leq c_{2} f_{1}(z)-c_{1} f_{2}(z) \quad(z \in \mathcal{Z}), \\
& c_{1} \leq\left\langle z_{0}, x\right\rangle \leq c_{2},
\end{aligned}
$$

where $z_{0}$ is given by $\chi_{S}$, the all-one vector in $\mathbb{R}^{S}$. Actually we will show that the pair $\left(f_{1}, f_{2}\right)$ for matroid intersection is not dual consistent in general.

Remark 8: In Section 4.2 we have seen that ordinary systems of linear inequalities for cardinality-constrained bipartite matchings are not dual consistent. However, this does not imply that the linear representations of the cardinality-constrained matroid intersection are not dual consistent in general, though the bipartite matching problem is a special case of the matroid intersection problem. Note that $\mathcal{Z} \supseteq 2^{S} \backslash\{\emptyset\}$ for matroid intersection and that this is not the case for ordinary bipartite matching polytopes. (We identify a subset of $S$ with its characteristic vector as before.)

Now let $\mathbf{M}^{(1)}$ and $\mathbf{M}^{(2)}$ be the graphic matroids on the ground set $S=\{1,2,3,4,5\}$ represented by the graphs $G_{1}$ and $G_{2}$ given in Figure 1.

Suppose $c_{1}=1$ and $c_{2}=4$. For an appropriately given weight vector $w$ we have

$$
I_{c_{1}}=\{5\}, \quad I_{c_{2}}=\{1,2,3,4\}
$$

as the unique maximum-weight common independent sets of size $c_{1}(=1)$ and $c_{2}(=4)$, respectively, which give the unique optimal solutions $\hat{x}_{1}=\chi_{I_{c_{1}}}$ and $\hat{x}_{2}=\chi_{I_{c_{2}}}$ of Problems 


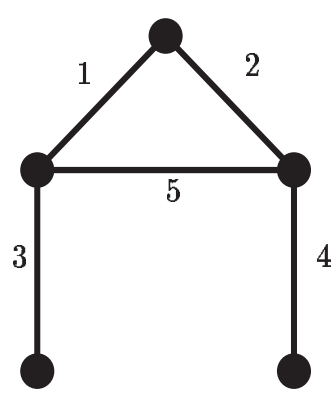

$G_{1}$

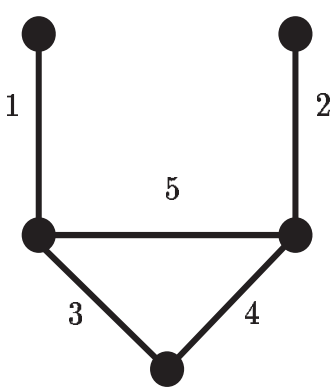

$G_{2}$

Figure 1: The graphs $G_{1}$ and $G_{2}$ representing the graphic matroids $\mathbf{M}^{(1)}$ and $\mathbf{M}^{(2)}$.

$\left(\mathbf{P}_{1}^{w}\right)$ and $\left(\mathbf{P}_{2}^{w}\right)$, respectively, due to the integrality of the matroid intersection polytope with a single cardinality constraint. For such a weight vector $w$ we can easily see that a common dual optimal base is given by the following five:

$$
S(=\{1,2,3,4,5\}), \quad S \backslash\{e\} \quad(e \in\{1,2,3,4\}) .
$$

Next, copy each of $\mathbf{M}^{(1)}$ and $\mathbf{M}^{(2)}$ on the ground set $S^{\prime}=\left\{1^{\prime}, 2^{\prime}, 3^{\prime}, 4^{\prime}, 5^{\prime}\right\}$. For $i=1,2$ consider the direct sum of $\mathbf{M}^{(i)}$ and its copy and denote it by $\mathbf{M}^{(i)}$ again, so that $\mathbf{M}^{(1)}$ and $\mathbf{M}^{(2)}$ are defined on the ground set $S \cup S^{\prime}=\left\{1,2,3,4,5,1^{\prime}, 2^{\prime}, 3^{\prime}, 4^{\prime}, 5^{\prime}\right\}$. Put $S \leftarrow S \cup S^{\prime}$ and let $c_{1}=2$ and $c_{2}=8$. For an appropriate weight vector $w$ we get $I_{c_{1}}=\left\{5,5^{\prime}\right\}$ as the unique maximum-weight common independent set of size $c_{1}(=2)$ and $I_{c_{2}}=\left\{1,2,3,4,1^{\prime}, 2^{\prime}, 3^{\prime}, 4^{\prime}\right\}$ as the unique maximum-weight common independent set of size $c_{2}(=8)$.

We can see that a maximum rank of common tight sets of $\hat{x}_{1}=\chi_{I_{c_{1}}}$ and $\hat{x}_{2}=\chi_{I_{c_{2}}}$ for the inequalities and equation in (6) for $i=1,2$ is attained by the following nine sets:

$$
\begin{aligned}
& S\left(=\left\{1,2,3,4,5,1^{\prime}, 2^{\prime}, 3^{\prime}, 4^{\prime}, 5^{\prime}\right\}\right), \\
& S \backslash\{e\} \quad e \in\left\{1,2,3,4,1^{\prime}, 2^{\prime}, 3^{\prime}, 4^{\prime}\right\} .
\end{aligned}
$$

Since there are ten variables, we do not have a common dual optimal base, i.e. the pair $\left(f_{1}, f_{2}\right)$ is not dual consistent. An additional valid inequality that yields a common dual base with respect to the present $w$ is given, for example, by

$$
6 x(\{5\})+x\left(\left\{1,2,3,4,5,1^{\prime}, 2^{\prime}, 3^{\prime}, 4^{\prime}, 5^{\prime}\right\}\right) \leq 8 .
$$


It is left open to give a finite set of additional inequalities in a systematic way that makes the systems for cardinality-constrained (poly)matroid intersection dual consistent.

It is conjectured in $[8,11]$ that the convex hull of $P_{f_{1}}^{c_{1}} \cup P_{f_{2}}^{c_{2}}$ is determined by

$$
\begin{aligned}
& x(U) \leq f(U) \quad(U \subseteq S), \\
& \left(c_{2}-c_{1}\right) x(U)-\left(f(U)-c_{1}\right) x(S) \leq c_{1}\left(c_{2}-f(U)\right) \\
& \quad\left(U \subseteq S \text { with } c_{1}<f(U)<c_{2}\right), \\
& c_{1} \leq x(S) \leq c_{2}, \quad x \geq \mathbf{0} .
\end{aligned}
$$

Similarly as discussed in Section 4.1 we can see that inequalities (41) are implied by inequalities (4) and (5), so that the polytope $\hat{P}$ determined by (4) and (5) is included in the polytope $P^{\prime}$ determined by (41). Since in our example the pair $\left(f_{1}, f_{2}\right)$ is not dual consistent, it follows from Theorem 1 that the convex hull $P_{f_{1}, f_{2}}^{c_{1}, c_{2}}$ of $P_{f_{1}}^{c_{1}} \cup P_{f_{2}}^{c_{2}}$ is strictly included in $\hat{P}$. Hence $P_{f_{1}, f_{2}}^{c_{1}, c_{2}} \neq P^{\prime}$ and our example given above disproves a conjecture of Maurras, Spiegelberg, and Stephan $[8,11]$ for the cardinality-constrained polymatroid intersection.

\section{Concluding Remarks}

We have introduced a new concept of dual consistency of systems of inequalities and have revealed that the concept of dual consistency plays a crucial role in the linear representation of cardinality constrained polytopes. We have also shown that the ordinary systems of inequalities for the cardinality-constrained bipartite matching polytopes are not dual consistent in general and have given a set of additional inequalities to make the system of inequalities dual consistent.

Moreover, we have shown that ordinary systems of inequalities for the cardinalityconstrained (poly)matroid intersection are not dual consistent in general, which disproves a conjecture of Maurras, Spiegelberg, and Stephan $[8,11]$ about a linear representation of the cardinality-constrained polymatroid intersection.

\section{References}

[1] P. Camion and J. Maurras: Polytopes à sommets dans l'ensemble $\{0,1\}^{n}$. Cahiers du Centre d'Études de Recherche Opérationnelle 24 (1982) 107-120.

[2] J. Edmonds: Submodular functions, matroids, and certain polyhedra. Proceedings of the Calgary International Conference on Combinatorial Structures and Their Applications (R. Guy, H. Hanani, N. Sauer and J. Schönheim, eds., Gordon and Breach, New York, 1970), pp. 69-87; also in: Combinatorial Optimization-Eureka, You 
Shrink! (M. Jünger, G. Reinelt and G. Rinaldi, eds., Lecture Notes in Computer Science 2570, Springer, Berlin, 2003), pp. 11-26.

[3] S. Fujishige: Dual greedy polyhedra, choice functions, and abstract convex geometries. Discrete Optimization 1 (2004) 41-49.

[4] S. Fujishige: Submodular Functions and Optimization (Second Edition), (Annals of Discrete Mathematics 58) (Elsevier, 2005).

[5] M. Grötschel: Cardinality homogeneous set systems, cycles in matroids, and associated polytopes. In: The sharpest cut. The impact of Manfred Padberg and his work (M. Grötschel, Ed., MPS-SIAM Series on Optimization 4, 2004), pp. 199-216.

[6] V. Kaibel and R. Stephan: On cardinality constrained cycle and path polytopes. Mathematical Programming, Ser. A, 123 (2010) 371-394.

[7] J. F. Maurras: An example of dual polytopes in the unit hypercube. Annals of Discrete Mathematics 1 (1977) 391-392.

[8] J. F. Maurras, I. Spiegelberg and R. Stephan: On cardinality constrained polymatroids. Discrete Applied Mathematics (2011), doi:10.1016/j.dam.2011.10.007 .

[9] J. F. Maurras and R. Stephan: On the cardinality constrained matroid polytope. Networks 57 (2011) 240-246.

[10] R. Stephan: Cardinality constrained combinatorial optimization: Complexity and polyhedra. Discrete Optimization 7 (2010) 99-113.

[11] R. Stephan and I. Spiegelberg: On cardinality constrained polymatroids. Electronic Notes in Discrete Mathematics 36 (2010) 1017-1024. 